\title{
MODELING AND COMPARING DEPENDENCIES IN MULTIVARIATE RISK PORTFOLIOS
}

\author{
BY \\ NiCOLE BÄUERLE \\ Abteilung Mathematik VII \\ Operations Research \\ Universität Ulm \\ AND \\ Alfred MÜller \\ Institut für Wirtschaftstheorie \\ und Operations Research \\ Universität Karlsruhe
}

\begin{abstract}
In this paper we investigate multivariate risk portfolios, where the risks are dependent. By providing some natural models for risk portfolios with the same marginal distributions we are able to compare two portfolios with different dependence structure with respect to their stop-loss premiums. In particular, some comparison results for portfolios with two-point distributions are obtained. The analysis is based on the concept of the so-called supermodular ordering. We also give some numerical results which indicate that dependencies in risk portfolios can have a severe impact on the stop-loss premium. In fact, we show that the effect of dependencies can grow beyond any given bound.
\end{abstract}

\section{KEYWORDS}

Dependent risks; Stop-loss premium; Supermodular order; Stop-loss order; Majorization; Comonotonicity; Exchangeable Bernoulli random variables

\section{INTRODUCTION}

In traditional risk theory for means of tractability, individual risks are usually assumed to be independent. Recent research has shown, however, that a positive dependence between risks leads to underestimation of the 
stop-loss premium for the aggregated loss. To the best of our knowledge, Heilmann (1986) and Hürlimann (1993) have been the first authors, who demonstrated the impact of dependencies on stop-loss premiums. More recently, Dhaene and Goovaerts (1996) investigated the effect of bivariate dependencies on the related stop-loss premium and gave an upper bound by determining the riskiest portfolio. Dhaene and Goovaerts (1997) made a first attempt to treat multivariate dependencies. They considered a special life insurance portfolio with two-point distributions. Their results were generalized by Müller (1997) who characterized the riskiest portfolio under all portfolios with equal marginals for arbitrary distributions. Wang (1997) suggested a set of tools for concrete modeling of dependencies in risk portfolios using the information given by the correlation coefficients.

In this paper we now propose some natural models for multivariate risk portfolios with different degree of dependence and same marginal distributions. The assumption about equal marginals is crucial here since our focus lies on comparing dependencies only. The results can of course be extended to unequal marginals by adding stochastic dominance. The models are defined in such a way that it is possible to compare two portfolios from the same class of models with respect to their stop-loss premiums. More precisely, we consider the classical individual model from risk theory, where the aggregate claim amount of a portfolio in a period is given by

$$
S=\sum_{i=1}^{n} X_{i}
$$

where $X_{i}$ is the random claim amount caused by policy $i, i=1, \ldots, n$. Throughout the paper we assume that the random variables $X_{i}$ are nonnegative with finite expectation. In a first model (model 3.1 in section 3) we assume that the risks can be divided into several groups, where each risk of a group is influenced by a global risk factor, a group specific risk factor and an individual risk factor. We show how the group structure of the portfolio affects the stop-loss premium and determine the safest and riskiest portfolio in this model class. On that occasion, we use the notion of majorization in order to compare the group structures.

In a second model (model 3.2 in section 3) we compare two portfolios, where both are subject to the same economic/physical environment, but the second portfolio contains an additional global risk factor which influences the risks of this portfolio in the same direction. Again, the marginal distributions are assumed to be equal for both portfolios. It can be proved that the stop-loss premium in the second scenario is greater than in the first one. This result is used later on to construct a portfolio, where the risks have two-point distributions and the portfolio can be characterized by a dependence parameter $\rho \in[0,1]$. The construction is such that increasing $\rho$ leads to a higher correlation in the portfolio and the two extreme cases 
$\rho=0$ and $\rho=1$ correspond to independence and comonotonicity respectively. We show that the stop-loss premium is increasing in the dependence parameter $\rho$.

In another model we compare portfolios which are given by exchangeable Bernoulli random variables. Here it can be shown that stop-loss order of the mixing distribution implies more riskiness for the aggregate claims. Moreover, in this setting, we prove that the ratio of the stop-loss premium in the riskiest scenario divided by the stop-loss premium of an arbitrary portfolio is increasing in the retention level.

Our models are very general and cover most of the specific parametric models considered by Wang (1997). There is one main difference between Wang's paper and this one. We mainly investigate, how dependencies affect the riskiness of portfolios, whereas Wang focuses on algorithms for simulation and efficient computation of concrete parametric models for correlated risks. Thus the two papers are complementary in so far as his algorithms for simulation can be easily adapted to our models.

Most of the comparison results we provide in this paper are based on the so-called supermodular ordering. This concept has recently proven to be valuable for comparing dependencies in random vectors in a wide range of applied probability models. For details see Bäuerle (1997a), Shaked and Shanthikumar (1997) and the references therein.

At the end of the paper we give a numerical example for model 3.1, which shows that dependencies can have a severe effect on the stop-loss premium. In particular we demonstrate that whenever the retention level exceeds the expected aggregate claim amount, the effect of dependence can be arbitrary worse.

The paper is organized as follows: section 2 contains some basic definitions and results about stochastic orderings and dependence which we will use in the sequel. Section 3 covers model 3.1 and 3.2 and section 4 is dedicated to the special case of risks with two-point distributions. The numerical results are summarized in section 5 .

\section{Stochastic Orderings and Dependence}

Let us first fix the notation. A portfolio of risks is a random vector $X=\left(X_{1}, \ldots, X_{n}\right)$ of $n$ individual risks, where an individual risk $X_{i}, 1 \leq i \leq n$ is a non-negative (univariate) random variable with a finite mean. For arbitrary univariate random variables $Y$ we denote the distribution function by $F_{Y}(t):=P(Y \leq t), t \in \mathbb{R}$ and $\bar{F}_{Y}(t):=P(Y>t)=1-F_{Y}(t)$ shall be the corresponding survival function. We will also frequently use the stop-loss transform $\pi_{Y}(t):=E(Y-t)^{+}=\int_{t}^{\infty} \bar{F}_{Y}(x) d x, t \in \mathbb{R}$. For a random vector $X=\left(X_{1}, \ldots, X_{n}\right)$ we similarly define the distribution function

$$
F_{X}(t):=P(X \leq t)=P\left(X_{1} \leq t_{1}, \ldots, X_{n} \leq t_{n}\right), \quad t=\left(t_{1}, \ldots, t_{n}\right) \in \mathbb{R}^{n}
$$


and the survival function

$$
\bar{F}_{X}(t):=P(X>t)=P\left(X_{1}>t_{1}, \ldots, X_{n}>t_{n}\right), t=\left(t_{1}, \ldots, t_{n}\right) \in \mathbb{R}^{n} .
$$

Note that for multivariate distributions in general $\bar{F}_{X}(t) \neq 1-F_{X}(t)$. If two random yariables or vectors $X$ and $Y$ have the same distribution, we will write $X \stackrel{d}{=} Y . X \sim F$ should be read as: $X$ has the distribution $F$.

Now we will introduce some stochastic order relations, which are wellknown concepts for comparing risks.

Definition 2.1 Let $X, Y$ be real random variables with finite means.

a) We say that $X$ precedes $Y$ in stochastic order, written $X \leq_{s t} Y$, if $F_{X}(t) \geq F_{Y}(t)$ for all $t \in \mathbb{R}$.

b) $X$ precedes $Y$ in stop-loss order, written $X \leq_{s l} Y$, if $\pi_{X}(t) \leq \pi_{Y}(t)$ for all $t \in \mathbb{R}$.

\section{Remarks:}

a) If $X \preceq Y$, where $\preceq$ may be any stochastic order relation, then we will also write $F_{X} \preceq F_{Y}$ whenever it is convenient.

b) If we have a family $F_{\theta}, \theta \in \Theta \subset \mathbb{R}$ of distributions, then we say that $F_{\theta}$ is stochastically increasing in $\theta$, if $F_{\theta} \leq_{s t} F_{\theta^{\prime}}$ for $\theta<\theta^{\prime}$.

c) Stop-loss order means, that the stop-loss reinsurance premium for the risk $Y$ is higher than that for $X$ for any retention $t$.

Now we collect some important properties of these orderings, which we will use frequently. They can be found e.g. in Shaked and Shanthikumar (1994) or Goovaerts et al. (1990).

\section{Theorem 2.2}

a) The following conditions are equivalent:

1. $X \leq_{s t} Y$,

2. $E f(X) \leq E f(Y)$ for all non-decreasing functions $f$,

3. There are random variables $\tilde{X} \stackrel{d}{=} X$ and $\tilde{Y} \stackrel{d}{=} Y$ such that $\tilde{X} \leq \tilde{Y}$ almost sure.

b) The following conditions are equivalent:

1. $X \leq_{s l} Y$,

2. $E f(X) \leq E f(Y)$ for all non-decreasing convex functions $f$,

3. There are random variables $\tilde{X} \stackrel{d}{=} X$ and $\tilde{Y} \stackrel{d}{=} Y$ such that $E[\tilde{Y} \mid \tilde{X}] \geq \tilde{X}$ almost sure.

As stated before, the main topic of this paper is the comparison of the riskiness of portfolios. In order to do so we need notions of stochastic order relations for random vectors. We say that a portfolio $X=\left(X_{1}, \ldots, X_{n}\right)$ is less risky than a portfolio $Y=\left(Y_{1}, \ldots, Y_{n}\right)$, if the corresponding aggregate claims $S=\sum_{i=1}^{n} X_{i}$ and $S^{\prime}=\sum_{i=1}^{n} Y_{i}$ are stop-loss ordered, i.e. $S \leq_{s l} S^{\prime}$. It will turn out that a sufficient condition for this is given by the so-called supermodular ordering or the symmetric supermodular ordering. These stochastic order relations have recently been considered in applied 
probability by Bäuerie (1997a, b), Bäuerle and Rieder (1997), Shaked and Shanthikumar (1997) and others. In the actuarial literature the supermodular ordering has been introduced by Müller (1997). It is based on the comparison of integrals of (symmetric) supermodular functions, which are defined as follows.

\section{Definition 2.3}

a) A function $f: \mathbb{R}^{n} \rightarrow \mathbb{R}$ is said to be supermodular, if

$$
\begin{aligned}
& f\left(x_{1}, \ldots, x_{i}+\varepsilon, \ldots, x_{j}+\delta, \ldots, x_{n}\right)-f\left(x_{1}, \ldots, x_{i}+\varepsilon, \ldots, x_{j}, \ldots, x_{n}\right) \\
& \geq f\left(x_{1}, \ldots, x_{i}, \ldots, x_{j}+\delta, \ldots, x_{n}\right)-f\left(x_{1}, \ldots, x_{i}, \ldots, x_{j}, \ldots, x_{n}\right)
\end{aligned}
$$

holds for all $x \in \mathbb{R}^{n}, 1 \leq i<j \leq n$ and all $\varepsilon, \delta>0$.

b) A function $f: \mathbb{R}^{n} \rightarrow \mathbb{R}$ is called symmetric, if $f(x)=f(\Pi x)$ for all permutations $\Pi x$ of $x$.

An intuitive explanation of the notion of supermodularity can be given as follows: Let $x_{1}, \ldots, x_{n}$ be the individual claim amounts of $n$ policy holders and let $f\left(x_{1}, \ldots, x_{n}\right)$ be the loss for the insurance company caused by these claims. Then supermodularity of the function $f$ means that the consequences of an increase of a single claim are the worse, the higher the other claims are.

Symmetric functions do not depend on the order of the variables. This means in our context that the policy holders are indistinguishable.

The following properties of supermodular functions are well-known.

\section{Theorem 2.4}

a) If $f$ is twice differentiable, then $f$ is supermodular if and only if

$$
\frac{\partial^{2}}{\partial x_{i} \partial x_{j}} f(x) \geq 0 \text { for all } x \in \mathbb{R}^{n}, 1 \leq i<j \leq n \text {. }
$$

b) If $g_{1}, \ldots, g_{n}: \mathbb{R} \rightarrow \mathbb{R}$ are increasing functions and $f$ is supermodular, then $f\left(g_{1}(\cdot), \ldots, g_{n}(\cdot)\right)$ is also supermodular.

A proof of this theorem and many examples can be found in Marshall and Olkin (1979, p. 146ff.). Now we will introduce the supermodular stochastic order relation.

\section{Definition 2.5}

a) $A$ random vector $X=\left(X_{1}, \ldots, X_{n}\right)$ is said to be smaller than the random vector $Y=\left(Y_{1}, \ldots, Y_{n}\right)$ in the supermodular ordering, written $X \leq_{s m} Y$, if $E f(X) \leq E f(Y)$ for all supermodular functions $f$ such that the expectations exist.

b) A random vector $X=\left(X_{1}, \ldots, X_{n}\right)$ is said to be smaller than the random vector $Y=\left(Y_{1}, \ldots, Y_{n}\right)$ in the symmetric supermodular ordering, written $X \leq_{\text {symsm }} Y$, if $E f(X) \leq E f(Y)$ for all symmetric supermodular functions $f$ such that the expectations exist. 
Supermodular ordering is a useful tool for comparing dependence structures of random vectors. Since any function $f: \mathbb{R}^{n} \rightarrow \mathbb{R}$ that depends only on one variable (i.e. $f\left(x_{1}, \ldots, x_{n}\right)=g\left(x_{i}\right)$ for some $g: \mathbb{R} \rightarrow \mathbb{R}$ and some $i \in\{1, \ldots, n\}$ ) is supermodular, it follows immediately from the definition that only distributions with the same marginals can be compared by supermodular ordering. Moreover, all functions $f(x)=x_{i} x_{j}, i \neq j$ are supermodular. Hence $X \leq_{s m} Y$ implies $\operatorname{Corr}\left(X_{i}, X_{j}\right) \leq \operatorname{Corr}\left(Y_{i}, Y_{j}\right), i \neq j$.

The usefulness of these concepts in our setting is shown clearly in the next result.

Theorem 2.6 Let $X=\left(X_{1}, \ldots, X_{n}\right)$ and $Y=\left(Y_{1}, \ldots, Y_{n}\right)$ be random vectors with $X \leq_{s m} Y\left(X \leq_{\text {symsm }} Y\right)$ and let

$$
S=\sum_{i=1}^{n} X_{i} \text { and } S^{\prime}=\sum_{i=1}^{n} Y_{i}
$$

Then $S \leq_{s l} S^{\prime}$.

Proof: For the supermodular ordering this has been shown in Müller (1997, Th. 3.1). The case of symmetric supermodular ordering can be shown along the same lines, as the function $x \rightarrow \sum x_{i}$ is obviously symmetric.

The Theorem says that stronger dependence in the sense of supermodular ordering leads to more risky portfolios. Next we will construct a special random vector with given marginals, which exhibits a very strong form of dependence. Let $U$ be a random variable uniformly distributed on $[0,1]$ and let $F_{1}, \ldots, F_{n}$ be $n$ marginal distributions. Define $X=\left(X_{1}, \ldots, X_{n}\right)=$ $\left(F_{1}^{-1}(U), \ldots, F_{n}^{-1}(U)\right)$. Using the well-known fact in simulation that $F^{-1}(U) \sim F$, we see that $X$ in fact has the marginal distributions $F_{1}, \ldots, F_{n}$. Since $F_{i}^{-1}$ is increasing for all $i$ it follows that $X_{i}\left(\omega_{1}\right)<X_{i}\left(\omega_{2}\right)$ implies $X_{j}\left(\omega_{1}\right) \leq X_{j}\left(\omega_{2}\right)$ for all $j \neq i$. Schmeidler (1986) and Yaari (1987) introduced the notion comonotonicity for this property. An easy calculation shows that the distribution function of $X$ is given by $F_{X}(t)=\min _{i=1}^{n} F_{i}\left(t_{i}\right)$. Summing up, we can give four equivalent definitions of comonotonicity.

Definition 2.7 The distribution $F$ with marginal distributions $F_{1}, \ldots, F_{n}$ is called comonotonic, if one of the following four equivalent conditions is fulfilled:

$$
F(t)=\min _{i=1}^{n} F_{i}\left(t_{i}\right), t \in \mathbb{R}^{n}
$$

2. The random vector $X=\left(F_{i}^{-1}(U), \ldots, F_{n}^{-1}(U)\right)$, where $U$ is uniformly distributed on $[0,1]$, has the distribution $F$,

3. There is a univariate random variable $Z$ and there are increasing functions $f_{1}, \ldots, f_{n}$, such that $X=\left(f_{1}(Z), \ldots, f_{n}(Z)\right)$ has the distribution $F$.

4. There is a random vector $X \sim F$, such that $X_{i}\left(\omega_{1}\right)<X_{i}\left(\omega_{2}\right)$ implies $X_{j}\left(\omega_{1}\right) \leq X_{j}\left(\omega_{2}\right)$ for all $j \neq i$. 
The comonotonic distribution $F$ is also called upper Fréchet bound, since Fréchet has shown that for any distribution function $G$ with marginals $F_{1}, \ldots, F_{n}$ we have $G \leq F$. An even stronger result is the so-called Lorentzinequality. It can be found e.g. as Theorem 5 in Tchen (1980) and can be stated as follows.

Theorem 2.8 Let $X$ be an arbitrary random vector and let $Y$ be the comonotonic random vector with the same marginals as $X$. Then $X \leq_{s m} Y$.

This means that comonotonicity is the strongest possible dependence structure and hence by Theorem 2.6 the corresponding portfolio is the riskiest one under all portfolios with the same marginals.

\section{THE MODELS}

In this section we consider several possibilites of modeling dependencies in risky portfolios. In our first model we assume that the portfolio consists of different groups, such that there is a strong dependence between the members of one group, but much less dependence between members of different groups. As a typical example where this is very realistic imagine a catastrophe risk like earthquakes or hurricanes, where the groups are specified by geographic regions. There is certainly a strong dependency between the expected losses of people from the same region, but the losses will be nearly independent for people who live far from each other. For such situations we suggest the following model. It was introduced by Tong (1989) and was further considered by Bäuerle (1997a).

\section{Model 3.1}

Consider a portfolio $X=\left(X_{1}, \ldots, X_{n}\right)$, consisting of $n$ risks $X_{1}, \ldots, X_{n}$. We assume that the risks can be divided into $r \leq n$ groups according to an $n$-dimensional vector $k=\left(k_{1}, \ldots, k_{r}, 0, \ldots, 0\right), k_{v} \in \mathbb{N}, \sum_{v=1}^{r} k_{v}=n$, where risk $X_{i}$ is in group $v$ if and only if $k_{1}+\ldots+k_{v-1}<i \leq k_{1}+\ldots+k_{v}$. Each of the risks in the portfolio is influenced by three risk factors which will be modeled as independent random variables $V, G_{v}$ and $Z_{i}$ :

1. an overall risk factor $V$ which is due to global environmental changes and concerns all of the risks in the portfolio in the same fashion,

2. a group specific risk factor $G_{v}$ which influences only the risks in group $v$, $1 \leq v \leq r$ and has no effect on other risks in the portfolio,

3. an individual risk factor $Z_{i}$ which reflects the individual share of risk $X_{i}$, $1 \leq i \leq n$.

Moreover, we assume that there exists a function $g: \mathbb{R}^{3} \rightarrow \mathbb{R}$ such that the $i$-th risk is given by $X_{i}=g\left(V, G_{v}, Z_{i}\right)$ whenever $i$ is in group $v$. Since we associate higher outcomes of a risk factor with higher risk in the portfolio, we suppose that $g$ is increasing. This situation is typical for a lot of insurance portfolios. In private health insurance for example, the risk caused by an individual person depends on an overall risk factor which collects 
environmental aspects (e.g. pollution, greenhouse effect, epidemics), on a group specific factor like profession and on an individual risk factor which summarizes health conditions. In car insurance, the group risk factor could be interpreted as the local area of the policy holder. Assuming this kind of dependence within a portfolio it is now interesting to investigate the effect, the constellation of group sizes has on the aggregate claim of the portfolio, since it is well-known that positive correlations in a risk portfolio increase the payable amount of the insurance company, see e.g. Dhaene and Goovaerts (1996, 1997) or Müller (1997). Obviously it is quite hard to compare two risky portfolios when for example the number and sizes of the groups change. However, in some cases this is possible as we will show in the next theorem. In order to state it, let $k$ and $k^{\prime}$ be two $n$-dimensional vectors with

$$
k=\left(k_{1}, \ldots, k_{r}, \ldots, 0, \ldots, 0\right), \quad k^{\prime}=\left(k_{1}^{\prime}, \ldots, k_{l}^{\prime}, 0, \ldots, 0\right)
$$

$1 \leq r, l \leq n, k_{i}, k_{i}^{\prime} \in N$ for all $i$ and $\sum_{i=1}^{n} k_{i}=\sum_{i=1}^{n} k_{i}^{\prime}=n$. Let two $n$-dimensional risky portfolios $X$ and $Y$ be given by

$$
\begin{array}{rlrl}
X_{1} & =g\left(Z_{1}, G_{1}, V\right) & Y_{1} & =g\left(U_{1}, G_{1}, V\right) \\
& \vdots & \vdots \\
X_{k_{1}} & =g\left(Z_{k_{1}}, G_{1}, V\right) & Y_{k_{1}^{\prime}} & =g\left(U_{k_{1}^{\prime}}, G_{1}, V\right) \\
X_{k_{1}+1} & =g\left(Z_{k_{1}+1}, G_{2}, V\right) & Y_{k_{1}^{\prime}+1} & =g\left(U_{k_{1}^{\prime}+1}, G_{1}, V\right) \\
& \vdots & \vdots \\
X_{k_{1}+k_{2}} & =g\left(Z_{k_{1}+k_{2}}, G_{2}, V\right) & Y_{k_{1}^{\prime}+k_{2}^{\prime}} & =g\left(U_{k_{1}^{\prime}+k_{2}^{\prime}}, G_{2}, V\right) \\
& \vdots & \vdots & \\
X_{n} & =g\left(Z_{n}, G_{r}, V\right) & Y_{n} & =g\left(U_{n}, G_{l}, V\right)
\end{array}
$$

where the individual risk factors $Z_{1}, \ldots, Z_{n}, U_{1}, \ldots, U_{n}$ are i.i.d. random variables, the group specific risk factors $G_{1}, \ldots, G_{\max \{r, l\}}$ are i.i.d. random variables and the environmental risk factor $V$ is a random variable independent of $\left\{Z_{i}\right\},\left\{U_{i}\right\}$ and $\left\{G_{v}\right\} . g: \mathbb{R}^{3} \rightarrow \mathbb{R}$ is an increasing function. Denote $S=\sum_{i=1}^{n} X_{i}$ and $S^{\prime}=\sum_{i=1}^{n} Y_{i}$ respectively.

Moreover, we need an appropriate order relation for vectors to compare the group structures $k$ and $k^{\prime}$. It turns out that the notion of majorization is best suited for this purpose. The definition is as follows.

Definition 3.1 Let $x, y \in \mathbb{N}_{0}^{n}$ and denote by $x_{[1]} \geq \ldots \geq x_{[n]}$ the decreasing rearrangement of $x$, analogously for $y$. We say that $y$ majorizes $x(x \prec y)$ if and only if

$$
\sum_{i=1}^{r} x_{[i]} \leq \sum_{i=1}^{r} y_{[i]}, \quad r=1, \ldots, n-1, \quad \text { and } \quad \sum_{i=1}^{n} x_{[i]}=\sum_{i=1}^{n} y_{[i]} .
$$


A host of results and applications of this order relation can be found in Marshall and Olkin (1979). Intuitively speaking $k \prec k^{\prime}$ means that in $k^{\prime}$ the groups are larger and/or more unequal. Some examples are given in section 5 . Now we are able to state the main result for this model.

Theorem 3.2 If $k \prec k^{\prime}$, we obtain under the assumptions of model 3.1

a) $X \leq_{\text {symsm }} Y$,

b) $S \leq_{s l} S^{\prime}$.

Proof: A complete proof of statement a) can be found in Bäuerle (1997a). The main ideas are as follows: in a first step we show that for a sequence $\left\{G_{\nu}\right\}$ of i.i.d. random variables and

$$
\begin{aligned}
X & =\left(G_{1}, \ldots, G_{1}, G_{2}, \ldots, G_{2}, \ldots, G_{r}, \ldots, G_{r}\right) \\
Y & =\left(G_{1}, \ldots, G_{1}, G_{2}, \ldots, G_{2}, \ldots, G_{l}, \ldots, G_{l}\right)
\end{aligned}
$$

where the block of $G_{i}$ 's in $X(Y)$ has length $k_{i}\left(k_{i}^{\prime}\right)$, the relation $k \prec k^{\prime}$ implies that $X \leq_{\text {symsm }} Y$. Applying properties of symmetric supermodular functions we obtain a). Part b) then follows from Theorem 2.6 .

In this setting it is easy to determine the riskiest and the safest portfolio with respect to the stop-loss ordering of aggregate claims. In order to do so we only need to determine the minimum and maximum with respect to majorization under all vectors $k$ with $\sum k_{i}=n$. It is nearly obvious that the minimum is given by $k^{s}:=(1,1, \ldots, 1)$ and the maximum is given by $k^{r}=(n, 0, \ldots, 0)$. This yields the following result.

Corollary 3.3 Let $k^{r}=(n, 0, \ldots, 0)$ and $k^{s}=(1, \ldots, 1)$ be two $n$-dimensional vectors and denote by $S^{r}$ and $S^{s}$ the aggregate claims of the corresponding risk portfolios as in model 3.1. Then we obtain for arbitrary $k \in \mathbb{N}_{0}^{n}$ with $\sum_{i=1}^{n} k_{i}=n$ and respective aggregate claim $S$

$$
S^{s} \leq_{s l} S \leq_{s l} S^{r}
$$

Hence the riskiest portfolio is given, when there is only one group and the safest portfolio is obtained, when each individual forms his/her own group.

Our model 3.1 is strongly related to the component models introduced in chapter 9 of Wang (1997). As another important class of models he considers common mixture models, which we will investigate now.

\section{Model 3.2}

The intuition behind this model is as follows. The model for $X$ as well as the model for $Y$ is a so called common mixture model. This means that there are some external mechanisms, described by random variables, which have influence on all the risks. Given these environmental parameters, the individual risks are independent. The parameters can be some state of nature (weather conditions, earthquakes, ...) as well as economic or legal 
environments (inflation, court rules etc.) which have a common impact on all risks. In contrast to model 3.1 we will now compare the portfolios with respect to the number of external mechanisms which affect them.

The following model for this situation has been considered by Bäuerle (1997a) (cf. also Shaked and Tong (1985)): Suppose there are two $n^{-}$ dimensional random vectors $X$ and $Y$ with the structure

$$
\begin{aligned}
& \left(X_{1}, \ldots, X_{n}\right)=\left(g_{1}\left(Z_{1}, W\right), \ldots, g_{n}\left(Z_{n}, W\right)\right) \\
& \left(Y_{1}, \ldots, Y_{n}\right)=\left(\tilde{g}_{1}\left(U_{1}, V, W\right), \ldots, \tilde{g}_{n}\left(U_{n}, V, W\right)\right)
\end{aligned}
$$

where $Z_{1}, \ldots, Z_{n}$ are i.i.d. random variables, $U_{1}, \ldots, U_{n}$ are i.i.d. random variables and $(V, W)$ is a random vector independent of $Z_{i}$ and $U_{i}$. Moreover, the functions $g_{i}: \mathbb{R}^{2} \rightarrow \mathbb{R}$ and $\tilde{g}_{i}: \mathbb{R}^{3} \rightarrow \mathbb{R}$ are such that for every fixed $w$ and all $i=1, \ldots, n$ we have

$$
g_{i}\left(Z_{i}, w\right) \stackrel{d}{=} \tilde{g}_{i}\left(U_{i}, V, w\right)
$$

i.e. they have the same distribution.

We will show now, that the portfolio $Y=\left(Y_{1}, \ldots, Y_{n}\right)$ is more risky than the portfolio $X=\left(X_{1}, \ldots, X_{n}\right)$, if the functions $\tilde{g}_{i}$ are increasing in the second argument. In fact, let $S:=\sum_{i=1}^{n} X_{i}$ and $S^{\prime}:=\sum_{i=1}^{n} Y_{i}$. Then the following holds.

Theorem 3.4 If the functions $\tilde{g}_{i}$ are increasing in the second argument, then

a) $X \leq_{s m} Y$,

b) $S \leq_{s l} S^{\prime}$.

Proof: a) can be found as Theorem 3.1 in Bäuerle (1997a). Part b) then follows immediately from a) by Theorem 2.6.

The model for $Y$ contains an additional environmental variable $V$, which has an influence on $Y_{1}, \ldots, Y_{n}$ in the same direction. Hence there is more dependence in $Y$ than in $X$, since the external mechanism, which has a common influence on all risk, is more important in $Y$. This will become more explicit in the special case we will treat now.

Let us assume that $W$ is constant. Hence $Y_{i}=\tilde{g}_{i}\left(U_{i}, V\right)$ and $X_{i}=g_{i}\left(Z_{i}\right)$. This means that $Y_{1}, \ldots, Y_{n}$ are conditionally independent given $V=v$ and the monotonicity of $\tilde{g}_{i}$ in the second argument means that the conditional distribution of $Y_{i}$ given $V=v$ is stochastically increasing in $v$ for all $i=$ $1, \ldots, n$. Moreover, $X_{1}, \ldots, X_{n}$ are independent random variables, which by (4) have the same marginal distributions as $Y_{1}, \ldots, Y_{n}$. Summing up, we get the following corollary of Theorem 3.4.

Corollary 3.5 Let $V$ be any random variable and let $Y=Y_{1}, \ldots, Y_{n}$ be a random vector such that $Y_{1}, \ldots, Y_{n}$ are conditionally independent given $V=v$ and such that the conditional distributions $P\left(Y_{i} \in \cdot V=v\right)$ are stochastically 
increasing in $v$ for all $i=1, \ldots, n$. Moreover, let $X=\left(X_{1}, \ldots, X_{n}\right)$ be a vector of independent random variables with the same marginal distributions as $Y$. Then

$$
X \leq_{s m} Y \quad \text { and } \quad S:=\sum_{i=1}^{n} X_{i} \leq_{s l} S^{\prime}:=\sum_{i=1}^{n} Y_{i}
$$

Another application of Theorem 3.4 will be given in the next section. Many more examples can be found in chapter 7 of Wang (1997).

\section{Risks With TWO-POINT DistRIBUTIONS}

Now we consider the important special case of portfolios consisting of risks $X_{i}$ having a two-point distribution in 0 and $\alpha_{i}$ with $P\left(X_{i}=0\right)=p_{i}$. This occurs e.g. in the individual life model. Dhaene and Goovaerts (1997) determined the riskiest portfolio with given marginals for this case and especially considered portfolios with dependencies only between couples.

The riskiest portfolio has the property that if a policy holder with a low mortality dies, then all policy holder with higher mortality also die with probability 1 . We think that this is very unrealistic. It would be desirable to have a parametric model with a dependence parameter $\rho$, which continuously varies between independence and maximal dependence as described above.

We investigate here two such models, one for the case of indistinguishable individuals and one for the case that the probability for no claim differs between the individuals.

\section{Indistinguishable individuals}

We say that the individuals in a portfolio are indistinguishable, if the joint distribution of the random vector of their risks is not affected by permutations of the risks. In probability theory a sequence of such random variables is said to be exchangeable (or interchangeable), see e.g. Feller (1966, p. 228ff.) or Chow and Teicher (1978). Of course this implies that all risks have the same marginal distribution, i.e. there is a $p \in(0,1)$ and some $\alpha>0$ such that $P\left(X_{i}=0\right)=p=1-P\left(X_{i}=\alpha\right)$ for all $i=1, \ldots, n$. Without loss of generality we can assume $\alpha=1$, so that the random variables $X_{1}, X_{2}, \ldots$ form a sequence of exchangeable Bernoulli variables.

Therefore let us assume that $S_{n}$ is the total claim amount of a portfolio of $n$ risks, which stem from a sequence of exchangeable Bernoulli variables. A well-known theorem of de Finetti (see e.g. Feller (1966, p. 228)) states that in this case $S_{n}$ is a mixture of binomial distributions, i.e.

$$
P\left(S_{n}=k\right)=\int_{0}^{1}\left(\begin{array}{l}
n \\
k
\end{array}\right) \vartheta^{k}(1-\vartheta)^{n-k} F(d \vartheta)
$$


for some mixing distributions $F$. Thus, the distribution of $S_{n}$ is completely determined by the mixing distribution $F$. In fact, it is completely determined by the first $n$ moments of $F$. For a survey on exchangeable Bernoulli variables, including many examples and methods for estimating their parameters we refer to Madsen (1993).

Now we want to show, how the mixing distribution $F$ affects the riskiness of the portfolio $S_{n}$. We have the following result.

Theorem 4.1 Let $S_{n}\left(S_{n}^{\prime}\right)$ be the total claim amount of a portfolio of $n$ risks, which stem from a sequence of exchangeable Bernoulli variables with mixing distribution $F\left(F^{\prime}\right)$. Then $F \leq_{s l} F^{\prime}$ implies $S_{n} \leq_{s l} S_{n}^{\prime}$.

Proof: This follows directly from Corollary 3.7 in Lefèvre and Utev (1996).

Remark: From Theorem 4.1 it follows easily that the least risky portfolio of exchangeable Bernoulli variables with given marginals is the one that consists of independent risks and the riskiest portfolio is the one with mixing distribution concentrated on $\{0,1\}$, which means that the risks are comonotonic. In fact, this means that the portfolio consists of identical risks $X=\left(X_{1}, X_{1}, \ldots, X_{1}\right)$ and the distribution of the total claim amount $S_{n}=n \cdot X_{1}$ is a two-point distribution with $P\left(S_{n}=0\right)=p=1-P\left(S_{n}=n\right)$. If we compare the stop-loss premiums of this portfolio with an arbitrary other portfolio of $b i(1, p)$-distributed risks, then we can strengthen Theorem 4.1 to the following result.

Theorem 4.2 Let $X=\left(X_{1}, \ldots, X_{n}\right)$ be a portfolio of bi $(1, p)$-distributed risks with an arbitrary dependence structure and let $Y=\left(Y_{1}, \ldots, Y_{1}\right)$ be a portfolio of identical risks with the same distribution. Let $\pi_{X}(t):=E\left(\sum X_{i}-t\right)^{+}$be the net stop-loss reinsurance premium of portfolio $X$ and define $\pi_{Y}(t)$ similarly. Then the ratio $\pi_{Y}(t) / \pi_{X}(t)$ is increasing on its range $[0, n)$.

Proof: Since $\sum Y_{i}=n Y_{1}$ is a two-point distribution on $\{0, n\}$, the function $\pi_{Y}$ is affine linear. Since any stop-loss transform is decreasing and convex (see e.g. Müller (1996)) this implies that $g(x):=\pi_{X} \circ \pi_{Y}^{-1}(x)$ is a convex function. Differentiation yields that

$$
g^{\prime}(x)=\frac{\pi_{X}^{\prime} \circ \pi_{Y}^{-1}(x)}{\pi_{Y}^{\prime} \circ \pi_{Y}^{-1}(x)}
$$

is increasing, and hence $\pi_{X}^{\prime}(x) / \pi_{Y}^{\prime}(x)$ is decreasing, since $\pi_{Y}^{-1}$ is decreasing. This can be written equivalently as

$$
\pi_{X}^{\prime}(t) \pi_{Y}^{\prime}(s) \geq \pi_{X}^{\prime}(s) \pi_{Y}^{\prime}(t) \quad \text { for all } t<s
$$


and hence

$$
\begin{aligned}
& \int_{t}^{\infty} \pi_{X}^{\prime}(t) \pi_{Y}^{\prime}(s) d s \geq \int_{t}^{\infty} \pi_{X}^{\prime}(s) \pi_{Y}^{\prime}(t) d s \\
& \Leftrightarrow \pi_{X}^{\prime}(t) \pi_{Y}(t) \leq \pi_{X}(t) \pi_{Y}^{\prime}(t) \\
& \Leftrightarrow \frac{\pi_{Y}(t)}{\pi_{X}(t)} \text { is increasing. }
\end{aligned}
$$

Remark: Computational results indicate that Theorem 4.2 may be true for arbitrary distributions. We are, however, not yet able to give a proof for this conjecture.

\section{Distinguishable individuals.}

Now we propose a model where the individuals in the portfolio may have different probabilities for claims and different claim amounts. We want to construct a portfolio of risks $X_{i}$ with $P\left(X_{i}=0\right)=p_{i}$ and $P\left(X_{i}=\alpha_{i}\right)=q_{i}=1-p_{i}$ where $0<p_{i}<1$ and $\alpha_{i}>0$ are arbitrary. Moreover we want to introduce a dependence parameter $\rho \in[0,1]$ such that $\rho=0$ corresponds to independence and $\rho=1$ corresponds to comonotonicity. A very simple model with this property would be to take some mixture of the independent and the comonotone case. We think, however, that this is not very realistic. We propose some sort of an additive damage model, which is well known in reliability theory. Assume that there are two sources, that cause some normally distributed damage. One source influences all individuals in the same manner, whereas the other source depends on the individual behavior of each individual. A claim of amount $\alpha_{i}$ occurs, if the sum of these two damages exceeds some level $z_{i}$.

The formal construction will be based on model 3.2 with distributions and functions, which assume only two values. We denote by $N\left(\mu, \sigma^{2}\right)$ the univariate normal distribution with mean $\mu$ and variance $\sigma^{2}>0$. For convenience we extend the definition to the case $\sigma^{2}=0$, where $N(\mu, 0)$ denotes the one-point distribution in $\mu$. The $p$-quantile of the standard normal distribution will be denoted by $z_{p}$, i.e. if $X \sim N(0,1)$, then $P\left(X \leq z_{p}\right)=p$. Now assume that $0 \leq \sigma^{2}<\tau^{2} \leq 1$ and consider model 3.2 with $W \sim N\left(0, \sigma^{2}\right), V \sim N\left(0, \tau^{2}-\sigma^{2}\right), Z_{i} \sim N\left(0,1-\sigma^{2}\right)$ and $U_{i} \sim N\left(0,1-\tau^{2}\right)$. All random variables shall be independent. We define

$$
g_{i}(z, w)=\alpha_{i} \cdot 1\left\{z+w \geq z_{p_{i}}\right\}:= \begin{cases}\alpha_{i}, & z+w \geq z_{p_{i}} \\ 0, & \text { else }\end{cases}
$$

and

$$
\tilde{g}_{i}(u, v, w)=\alpha_{i} \cdot 1\left\{u+v+w \geq z_{p_{i}}\right\} .
$$


Recall that $X_{i}=g_{i}\left(Z_{i}, W\right)$ and $Y_{i}=\tilde{g}_{i}\left(U_{i}, V, W\right)$ for $i=1, \ldots, n$. Since $U_{i}+V \stackrel{d}{=} Z_{i} \sim N\left(0,1-\sigma^{2}\right)$, condition (4) is fulfilled. Moreover, $Z_{i}+W \stackrel{d}{=} U_{i}+V+W \sim N(0,1)$, so that $P\left(X_{i}=\alpha_{i}\right)=P\left(Z_{i}+W \geq z_{p_{i}}\right)=q_{i}$ and $P\left(X_{i}=0\right)=P\left(Z_{i}+W \leq z_{p_{i}}\right)=p_{i}$. Similarly $P\left(Y_{i}=0\right)=p_{i}=1-P\left(Y_{i}=\alpha_{i}\right)$. By Theorem $3.4 X \leq_{s m} Y$ and hence $X$ is less risky than $Y$.

Now let us write $X(\sigma)=\left(X_{1}(\sigma), \ldots, X_{n}(\sigma)\right)$ for the above defined portfolio $X$ to make the dependency on $\sigma$ explicit. The definition of $Y$ implies that $Y \stackrel{d}{=} X\left(\tau^{2}\right)$ which can be seen by interchanging the roles of $Z_{i}$ and $U_{i}$ as well as the one of $W$ and $V+W$. Hence we obtain the following result.

Theorem 4.3 Let $0 \leq \rho<\rho^{\prime} \leq 1$. Then $X(\rho) \leq_{s m} X\left(\rho^{\prime}\right)$ and hence

$$
\sum_{i=1}^{n} X_{i}(\rho) \leq_{s I} \sum_{i=1}^{n} X_{i}\left(\rho^{\prime}\right) \text {. }
$$

It is easy to see that $X(0)$ is a portfolio of independent risks and $X(1)$ is a portfolio of comonotonic risks, which is the riskiest portfolio under all portfolios with given marginals, as has been shown by Müller (1997) for general distributions and in Dhaene and Goovaerts (1997) for the case of two-point distributions as considered here. Now we will show that we can get any positive dependence structure by varying $\rho$ continuously between these two extreme cases. In fact, we have the following result.

Theorem 4.4 The function $\rho \rightarrow \operatorname{Corr}\left(X_{i}(\rho), X_{j}(\rho)\right)$ is non-negative and continuously increasing for all $i, j=1, \ldots, n, i \neq j$.

Proof: The marginal distribution of $X_{i}(\rho)$ and hence also the variance of $X_{i}(\rho)$ is independent of $\rho$ for $i=1, \ldots, n$. Thus we only have to examine the covariance. A straightforward calculation shows that

$$
\operatorname{Cov}\left(X_{i}(\rho), X_{j}(\rho)\right)=\alpha_{i} \alpha_{j} \cdot\left(P\left(X_{i}(\rho)=\alpha_{i}, X_{j}(\rho)=\alpha_{j}\right)-q_{i} q_{j}\right) .
$$

Hence it is sufficient to consider the expression

$$
P\left(X_{i}(\rho)=\alpha_{i}, X_{j}(\rho)=\alpha_{j}\right)=P\left(Z_{i}+W \geq z_{p_{i}}, Z_{j}+W \geq z_{p_{j}}\right)=: \bar{F}_{\rho}\left(z_{p_{i}}, z_{p_{j}}\right)
$$

where $\bar{F}_{\rho}$ is the survival function of a bivariate normal distribution with standard normal marginals and correlation coefficient $\rho$. It follows from Slepian's inequality and its proof as given e.g. in Tong $(1980$, p. 8ff.) that $\rho \rightarrow \bar{F}_{\rho}$ is increasing and continuous. Hence $\rho \rightarrow \operatorname{Corr}\left(X_{i}(\rho), X_{j}(\rho)\right)$ is also increasing and continuous. Non-negativity then follows from the fact that $X(0)$ is a vector of independent random variables. 


\section{Numerical EXAMPLE}

Let us now illustrate the effect of dependencies in model 3.1 by a numerical example. In order to keep the computation simple, we have chosen $g(x, y, z)$ $=y$. The sequence of random variables $\left\{G_{v}\right\}$ is i.i.d. with a two-point distribution on 0 and 4 , where the value of 4 occurs with probability 0.06 . The portfolio consists of 20 risks. We have computed the relative stop-loss premiums for 8 different scenarios which are given by their group structures $k_{i}, i=1, \ldots, 8$ listed in table 1 .

TABLE 1

\begin{tabular}{c|c}
\hline scenario $i$ & $k_{i}$ \\
\hline 1 & $(1,1,1, \ldots, 1,1,1)$ \\
2 & $(4,3,3,2,2,1,1,1,1,1,1)$ \\
4 & $(8,2,2,2,2,2,2)$ \\
5 & $(4,4,4,3,3,2)$ \\
6 & $(15,2,1,1,1)$ \\
7 & $(5,5,5,5)$ \\
8 & $(10,5,5)$ \\
-
\end{tabular}

Scenario 1 corresponds to the safest portfolio with 20 independent risks and scenario 8 is the riskiest portfolio, where the same risk occurs 20 times. In the next table we summarize the ordering relations of these vectors with respect to the majorization ordering.

TABLE 2

\begin{tabular}{l|cccccccc}
\hline & $k_{1}$ & $k_{2}$ & $k_{3}$ & $k_{4}$ & $k_{5}$ & $k_{6}$ & $k_{7}$ & $k_{8}$ \\
\hline$k_{1}$ & $\prec$ & $\prec$ & $\prec$ & $\prec$ & $\prec$ & $\prec$ & $\prec$ & $\prec$ \\
$k_{2}$ & & $\prec$ & $\prec$ & $\prec$ & $\prec$ & $\prec$ & $\prec$ & $\prec$ \\
$k_{3}$ & & & $\prec$ & $\nprec$ & $\prec$ & $\nprec$ & $\prec$ & $\prec$ \\
$k_{4}$ & & & & $\prec$ & $\prec$ & $\prec$ & $\prec$ & $\prec$ \\
$k_{5}$ & & & & & $\prec$ & $\nprec$ & $\prec$ & $\prec$ \\
$k_{6}$ & & & & & & $\prec$ & $\prec$ & $\prec$ \\
$k_{7}$ & & & & & & & $\prec$ & $\prec$ \\
\hline
\end{tabular}

The symbol $\nprec$ indicates that the vectors cannot be compared. The following table now contains the relative stop-loss premiums (divided by the independent case $i=1$ ) multiplied by 100 for several retention levels. Note that the expectation of the aggregate claims equals 4.8 and the outcomes range between 0 and 80 . 
TABLE 3

\begin{tabular}{c|cccccccc}
\hline & \multicolumn{10}{c}{ scenario } \\
\hline retention & $k_{1}$ & $k_{2}$ & $k_{3}$ & $k_{4}$ & $k_{5}$ & $k_{6}$ & $k_{7}$ & $k_{8}$ \\
\hline 0 & 100 & 100 & 100 & 100 & 100 & 100 & 100 & 100 \\
1 & 100 & 105 & 109 & 110 & 111 & 112 & 113 & 116 \\
2 & 100 & 113 & 121 & 124 & 126 & 129 & 132 & 139 \\
3 & 100 & 124 & 140 & 145 & 150 & 155 & 161 & 173 \\
4 & 100 & 144 & 173 & 182 & 191 & 200 & 210 & 233 \\
6 & 100 & 174 & 210 & 229 & 272 & 272 & 295 & 347 \\
8 & 100 & 270 & 330 & 385 & 537 & 506 & 572 & 717 \\
10 & 100 & 327 & 478 & 480 & 830 & 700 & 834 & 1128 \\
\hline \hline
\end{tabular}

Because of Theorem 3.2 we know that given a retention level, the relative stop-loss premium increases in $k$. Table 3 shows that the increase is moderate if $k_{i}$ and $k_{j}$ are in some sense nearby as for example $k_{6}$ and $k_{7}$. In the cases where we were not able to establish the comparison theoretically like for example for scenario 5 and 6 , we find that the order can change when the retention level increases. Theorem 4.2 explains the monotonicity of the relative stop-loss premium with respect to the retention in scenario 8 . The numerical data suggest that this is also true for the other scenarios. This was already observed by Dhaene and Goovaerts (1996). To our knowledge this is still an open problem.

A very important conclusion that we can draw from the computation is that the increase in the relative stop-loss premium can be dramatic in the presence of positive dependence. Even minor occurrence of dependence like in scenario 2 has a severe effect. Moreover, if a portfolio contains positive dependence between the risks, the situation deteriorates in the number of insured risks.

Suppose $Y, X_{1}, \ldots, X_{n}$ are i.i.d. random variables (w.l.o.g. we assume that they are concentrated on $[0,1])$ and we are interested in the stop-loss premiums of the safest portfolio $\pi_{X}^{n}(t)=E\left(\sum_{i=1}^{n} X_{i}-n t\right)^{+}$and the riskiest one $\pi_{Y}^{n}(t)=E(n Y-n t)^{+}$, where $t \in(0,1)$ gives the retention percentage. In this setting we obtain

Theorem 5.1 The ratio $\pi_{Y}^{n}(t) / \pi_{X}^{n}(t)$ is increasing in the number $n$ of aggregate risks and the limit is equal to $E(Y-t)^{+} /(E Y-t)$ if $t<E Y$ and $+\infty$ if $t \geq E Y$.

Proof: We obtain that

$$
\frac{\pi_{Y}^{n}(t)}{\pi_{X}^{n}(t)}=\frac{E(n Y-n t)^{+}}{E\left(\sum_{i=1}^{n} X_{i}-n t\right)^{+}}=\frac{E(Y-t)^{+}}{E\left(\frac{1}{n} \sum_{i=1}^{n} X_{i}-t\right)^{+}}
$$


Hence it suffices to prove that $E\left(\frac{1}{n} \sum_{i=1}^{n} X_{i}-t\right)^{+}$is decreasing in $n$. Since $X_{1}, \ldots, X_{n}$ are i.i.d. it follows from Theorem 4 in Arnold and Villasenor (1986) that

$$
\frac{1}{n+1} \sum_{i=1}^{n+1} X_{i} \leq_{s l} \frac{1}{n} \sum_{i=1}^{n} X_{i}
$$

and the monotonicity follows.

Since the random variables $X_{1}, X_{2}, \ldots$ are independent and identically distributed with a finite mean, the assumptions of the strong law of large numbers are fulfilled. Therefore

$$
\lim _{n \rightarrow \infty} \frac{1}{n} \sum_{i=1}^{n} X_{i}=E X_{1}=E Y .
$$

Hence the stated limit follows.

Remark: Arnold and Villasenor (1986) have shown that for Equation 5 it is sufficient, that $X_{1}, X_{2}, \ldots$ are exchangeable. Hence the monotonicity part of Theorem 5.1 remains true for the more general case of exchangeable random variables, but in that case the limit will be different. In fact, there is also a version of the strong law of large numbers for sequences of exchangeable random variables. It states that in this case

$$
\lim _{n \rightarrow \infty} \frac{1}{n} \sum_{i=1}^{n} X_{i}=E\left[X_{1} \mid \Theta\right]
$$

where $\Theta$ is the random variable, which describes the mixing mechanism in de Finetti's Theorem (cf. Feller (1966) and Chow and Teicher (1978) for more details). Hence in this case we get

$$
\lim _{n \rightarrow \infty} \frac{\pi_{Y}^{n}(t)}{\pi_{X}^{n}(t)}=\frac{E(Y-t)^{+}}{E(E[Y \mid \Theta]-t)^{+}}
$$

From Theorem 5.1 we see that the relative stop-loss premium can be arbitrary high, when the retention exceeds the expected aggregate claim. Altogether we can conclude that the usual assumption of independence in risky portfolios leads to a dangerous underestimation of the risk. Hence the adequate modeling of dependent risks will remain an important task for future research.

\section{ACKNOWLEDGEMENTS}

We would like to thank the anonymous referees for several helpful comments. 


\section{REFERENCES}

ARNOLD, B.C., J.A. VillaAsenOR (1986). Lorenz ordering of means and medians. Statistics and Probability Letters 4, 47-49.

BÄUERLE, N. (1997a). Inequalities for stochastic models via supermodular orderings. Communications in Statistics - Stochastic Models 13, 181-201.

BÄUERLE, N. (1997b). Monotonicity results for MR/GI/1 queues. Journal of Applied Probabiiity 34, $514-524$.

BÄUerLe, N., U. RIEDER (1997). Comparison results for Markov-modulated recursive models. Probability in the Engineering and Informational Sciences 11, 203-217.

Chow, Y.S., H. Teicher (1978). Probability Theory. Springer, New York.

DhaEne, J., M.J. GoovaerTs (1996). Dependency of risks and stop-loss order. ASTIN Bulletin 26, 201-212.

Dhaene, J., M.J. Goovaerts (1997). On the dependency of risks in the individual life model. Insurance: Mathematics and Economics 19, 243-253.

Feller, W. (1966). An introduction to Probability Theory and Its Applications, Volume II. Wiley, New York.

Goovaerts, M.J., R. KaAs, A.E. van Heerwaarden, T. Bauwelinckx (1990). Effective Actuarial Methods. Insurance Series vol. 3, North Holland.

HeIlmanN, W.-R. (1986). On the impact of independence of risks on stop loss transforms. Insurance: Mathematics and Economics 5, 197-199.

HÜrLIMANN, W. (1993). Bivariate distributions with diatomic conditionals and stop-loss transforms of random sums. Statistics and Probability Letters 17, 329-335.

LefÉvre, C., S. Utev (1996). Comparing sums of exchangeable Bernoulli random variables. Journal of Applied Probability 33, 285-310.

Madsen, R.W. (1993). Generalized binomial distributions. Communications in Statistics Theory and Methods 22, 3065-3086.

Marshall, A.W., I. OlKIN (1979). Inequalities: Theory of Majorization and its Applications. Academic Press, New York.

Müller, A. (1996). Orderings of risks: A comparative study via stop-loss transforms. Insurance: Mathematics and Economics 17, 215-222.

Müller, A. (1997). Stop-Loss Order for Portfolios of Dependent Risks. Insurance: Mathematics and Economics 21, 219-223.

SChmeIDleR, D. (1986). Integral Representation without additivity. Proceedings of the American Mathematical Society 97, 255-261.

Shaked, M., J.G. Shanthikumar (1994). Stochastic Orders and their Applications. Academic Press, London.

Shaked, M., J.G. Shanthikumar (1997). Supermodular Stochastic Orders and Positive Dependence of Random Vectors. Journal of Multivariate Analysis 61, 86-101.

Shaked, M., Y.L. Tong (1985). Some Partial Orderings of Exchangeable Random Variables by Positive Dependence. Journal of Multivariate Analysis 17, 333-349.

TCHEN, A.H. (1980). Inequalities for Distributions with given marginals. Annals of Probability 8, 814-827.

TONG, Y.L. (1980). Probability Inequalities in multivariate Distributions. Academic Press, New York.

TONG, Y.L. (1989). Inequalities for a class of positively dependent random variables with a common marginal. Annals of Statistics 17, 429-435.

WaNG, S. (1997). Aggregation of Correlated Risk Portfolios: Models and Algorithms. Preprint. YAARI, M.E. (1987). The dual theory of choice under risk. Econometrica 55, 95-115.

NiCOLE BäUERLE

Abteilung Mathematik VII

(Operations Research)

Helmholtzstr. 18

Universität Ulm

D-89069 Ulm, Germany
Alfred MüLleR

Institut für Wirtschaftstheorie und

Operations Research

Kaiserstr. 12

Universität Karlsruhe

D-76128 Karlsruhe, Germany 